\title{
Early Outcomes of Pelvic Trauma Patients - Complications, ICU Stay and Treatment Concepts in Two ATLS Compliant Trauma Centers: Germany and Qatar
}

\section{Holger Keil}

Universitatsklinikum Erlangen https://orcid.org/0000-0002-5006-8420

\section{Ayman El-Menyar}

Hamad General Hospital

Julian Daerr

BG Klinik Ludwigshafen

Frederik Weil

BG Klinik Ludwigshafen

Husham Abdelrahman

Hamad General Hospital

Abduljabbar Alhammoud

Hamad General Hospital

Syed I Ghouri

Hamad General Hospital

\section{Bianca M Wahlen}

Hamad General Hospital

Hassan Al-Thani

Hamad General Hospital

\section{Paul A Grützner}

BG Klinik Ludwigshafen

Matthias Münzberg ( $\square$ matthias.muenzberg@bgu-ludwigshafen.de)

BG Klinik Ludwigshafen

Original research

Keywords: ATLS®, pelvic injury, complications, polytrauma

Posted Date: December 30th, 2020

DOI: https://doi.org/10.21203/rs.3.rs-135818/v1 
License: (c) (i) This work is licensed under a Creative Commons Attribution 4.0 International License. Read Full License 
Early outcomes of pelvic trauma patients - complications, ICU stay and treatment concepts in two ATLS compliant trauma centers: Germany and Qatar

Holger Keil ${ }^{1,2}$, Ayman El-Menyar ${ }^{3,4}$, Julian Daerr ${ }^{2}$, Frederik Weil' ${ }^{2}$, Husham Abdelrahman ${ }^{5}$, Abduljabbar Alhammoud ${ }^{6}$, Syed I. Ghouri ${ }^{6}$, ElHadi Babikir ${ }^{6}$, Bianca M.Wahlen ${ }^{5}$, Hassan AlThani $^{5}$, Paul A. Gruetzner ${ }^{2}$, Matthias Muenzberg ${ }^{2 *}$

1 Universitätsklinikum Erlangen

Trauma and Orthopedic Surgery

Krankenhausstr. 12

91054 Erlangen

Germany

${ }^{2}$ BG Trauma Center

Ludwig-Guttmann-Str. 13

67071 Ludwigshafen

Germany

${ }^{3}$ Trauma Surgery, Clinical Research, Hamad General Hospital. Doha, Qatar

${ }^{4}$ Clinical Medicine , Weill Cornell Medical School, Doha, Qatar

5 Trauma Surgery, Hamad General Hospital. Doha, Qatar

${ }^{6}$ Orthopedic Surgery, Hamad General Hospital. Doha, Qatar

${ }^{*}$ Corresponding Author:

Matthias Muenzberg

BG Trauma Center

Ludwig-Guttmann-Str. 13

67071 Ludwigshafen

Germany

Email: matthias.muenzberg@bgu-ludwigshafen.de

Finiancial disclosure: none

Conflicts of interest: none

Funding: none

Keywords

ATLS $\AA$, pelvic injury, complications, polytrauma 


\title{
Early outcomes of pelvic trauma patients - complications, ICU stay and treatment concepts in two ATLS compliant trauma centers: Germany and Qatar
}

\begin{abstract}
Background: Pelvic trauma causes severe threats especially to polytraumatized patients. Not only it is in itself a possible cause for significant bleeding but it also indicates a high risk for intra-abdominal injuries. The initial treatment of patients with pelvic trauma follows the ATLS principles of priority-oriented treatment. To examine the value of this highly standardized concept and to evaluate the effect of different patient collectives on early outcome parameters, two large collectives from Germany and Qatar were analyzed regarding injury parameters and early outcomes.
\end{abstract}

Methods: Patients were recruited in Hamad General Hospital, Doha, Qatar (HGH) and BG Trauma Center Ludwigshafen, Germany (BG). All patients that were treated with a pelvic fracture between 2013 and 2016 were included in this retrospective analysis. Demographic parameters were collected as well as type of injury and the frequency of complication parameters as pneumonia, acute kidney failure, ARDS, sepsis and amount of blood transfusion.

Results: We recruited 1436 patients with pelvic fracture (645 from BG and 791 from HGH). The mean age was 57.4 years in the $B G$ and 33.6 years in the HGH group $(p<0.000)$. The mean ISS was 17.81 in the BG and 15.88 in the HGH group $(p=0.009)$. The mean pelvic AIS was 2.65 in the $B G$ and 2.25 in the HGH group $(p<0.000)$. The mean frequency of complications was $9.3 \%$ in the $B G$ and $9.9 \%$ in the HGH group $(p=0.128)$. The mean frequency of ARDS was significantly higher in the BG group than in the HGH group $(5.6 \%$ vs. $1.8 \%, p<0.000)$. The mean frequency of blood transfusion was significantly lower in the BG group than in the HGH group $(28.8 \%$ vs. $39.2 \%, p<0.000)$.

Conclusions: Despite significant differences in the two collectives, this analysis shows comparable results regarding early outcome parameters in patients with pelvic injuries. In total, pelvic injuries are accompanied by a relatively high complication risk and need to be evaluated and treated according to priority-based algorithms. 


\section{Background}

Although pelvic fractures make up only $2-3 \%$ of all fractures, they regularly pose high demands for institutions and surgeons treating these injuries. Despite advances in injury prevention as well as diagnostics and treatment strategies, pelvic trauma remains a challenging entity due to high association of significant abdominal and vascular injuries as well as high potential for massive retroperitoneal hemorrhage. In polytraumatized patients, mortality of pelvic trauma is high, reported up to $45 \%$. Driven by a high morbidity and mortality after severe trauma, American College of Surgery developed a priority-orientated diagnostic and treatment strategy for the first hours after trauma called ATLS (Advanced Trauma Life Support) [1]. ATLS principles were introduced in the 1970s and was steadily evolved from then. In ATLS courses doctors are taught a structured approach to address the immediate life-threatening trauma quickly by diagnosing the underlying causes for hemodynamic instability. Pelvic fracture is one of the main sources for massive blood loss. In ATLS principles, diagnosis and initial treatment of unstable pelvic fractures have a central role in managing circulation disorders ("C-Problems"). Diagnosing a pelvic fracture is possible through clinical examination and available radiography. Elements of initial care are giving blood transfusions and stabilization of the pelvic (external or internal) in order to minimize the blood loss by establishing stability and reducing the pelvic volume. The ATLS concept is widely adapted in Level-1 Trauma Centers worldwide, standardizing approaches and enabling hospitals and staff to treat such injuries similarly - regardless of the geographic location. With its principles ATLS is practiced transnationally.

To evaluate the efficacy of standardized concepts in different settings; in this study large collection of pelvic injuries were compared between a Level-1 Trauma center in a German urban area (BG Trauma Center Ludwigshafen; BG group) and a Qatari Level-1 center in the metropolitan area of Doha (Hamad General Hospital, Doha; HGH group). The wider urban area of Ludwigshafen in Germany covers around 700,000 citizens in comparison to 590,000 in Doha, Qatar. Both countries belong to high income economies. Beside the political system there are big differences in demographics, migration, religion, health system and infrastructure between Ludwigshafen and Doha. The Department of Economic and Social Affairs of the United Nations reports regularly about migration all over the world. It is described that Qatar is with an average annual rate of change in the number of international migrants over $6 \%$ one of three countries with the highest rate between 2000 and 2017. In contrast, Germany was one of 35 countries with an average annual rate of change in the number of international migrants less than $2 \%$. Next to the differences in migration where Qatar has a high percentage of expatriates (which are typically young males) and thus, there are large differences in demographic profiles of the two countries. there is an imbalance in the ratio of female and male citizens in Qatar, the main population is made up by young people aged 20-40 while in Germany, the age distribution is more homogenous from ages 20-70 with a peak between $50-60$ years.

Beside the differences in society, there are differences in the health system as well. In Qatar, health care is free for locals and financed by the state. In Germany there is a mandatory health care insurance established. The financing of costs in health care is given by social security and social health insurance.

In conclusion both health care systems compete with totally different circumstances. Nevertheless, we hypothesized that, despite the previously mentioned differences between Germany and Qatar, a standardized approach for managing trauma associated injuries like the ATLS being used in both countries, that there are no relevant differences in morbidity and mortality of pelvic injury.

\section{Methods}

A retrospective review of the registry of patients with pelvic trauma treated and followed up at the trauma department at Hamad Medical Corporation, Doha, Qatar, and BG Trauma Center Ludwigshafen, Germany (BG) from January 2013 to December 2017. The local ethics committee was addressed and gave consent to this study (BG 837.500.17, HMC 16395/16). In both centers, all patients that were treated between 2013 and 2016 and met the following inclusion criteria were included: 
- $\quad$ Pelvic trauma with clinical suspiciency of a fracture

- $\quad$ admitted and managed in the respective center

We collected data on demographic variables (age, gender, nationality), mechanism of injury (fall from height, road traffic accident, rollover trauma), trauma-associated factors (ISS Injury Severity Score, associated injuries, severity of injury (AIS - Abbreviated Injury Score), classification of the fracture (AO), management plan (operative, conservative), and early complications (ventilator-associated pneumonia (VAP)), acute respiratory distress syndrome (ARDS), acute kidney failure and sepsis, length of stay (overall hospital and ICU), ventilator days and blood transfusion.

A standard treatment protocol was used in all cases in both centers, which included: ATLS protocol, pan CT, admission in ICU or ward, orthopedics consultation and intervention. Statistical analysis was done using the Statistical Package for the Social Sciences (SPSS 24) (IBM, Armonk, USA). For estimating the differences of the occurrence of early complications, statistical analysis using the chi square test. For variables with normal distribution, Student's t-test was used. Statistical significance was assumed when $p<0.05$.

\section{Results}

To compare the effect of early diagnosing and treatment after trauma in the way of ATLS on complication rate and mortality first of all two patient collectives called BG group and HGH group were compared. A total of 1436 patients were recruited (645 patients of the BG group and 791 patients of the HGH group). The descriptive data is shown in Table 1.

For comparison of the trauma severity, the abbreviated injury scale (AIS) for pelvic injury was examined. The higher number of B- and C-type injuries in the BG group reflects in a statistically significant higher average AIS pelvis in the BG group (2.65 vs. $2.25, p<0.000)$. The differences in AIS pelvis are drawn in Figure 1.

Furthermore, the Injury Severity Score (ISS) in total was examined. The comparison of the ISS of patients of the BG group with patients of the HGH group showed no statistically significant difference (17.81 vs. $15.88, p=0.067$ ). In the $B G$ group $36.9 \%$ of the patients had an ISS > 16, in the HGH group it was $41.3 \%$. In general, especially younger male patients had a higher severity in trauma with a higher ISS than female patients (17.42 vs. 14.88, $\mathrm{p}<0.000)$. The differences in the ISS are shown in Figure 2.

\section{Differences in early complications after pelvic injury}

There was no significant difference in the mean frequency of complications between the BG and $\mathrm{HGH}$ group. A complications rate of $9.3 \%$ could be found in the BG group and one of $9.9 \%$ in the HGH group ( $p=0.128$ ). Divided in the certain complications, the mean frequency of ARDS was significantly higher in the BG group than in the HGH group (5.6\% vs. $1.8 \%$, $\mathrm{p}<0.000$ ). There was no significant difference in the occurrence of VAP (BG group: $4.8 \%$ vs. HGH group $7.1 \%, p=0.072)$, neither in the occurrence of acute kidney failure (3.4\% vs. $2.5 \%$, $p=0.324)$ nor in the occurrence of sepsis $(3,1 \%$ vs. $3,3 \%, p=0,842)$. The mean frequency of blood transfusion was significantly lower in the BG group than in the HGH group $(28.8 \%$ vs. $39.2 \%, p<0.000)$. The median number of transfused units per affected patient was significantly higher in the BG group ( 6 vs. $4, \mathrm{p}<0.000$ ). Mortality was low in both centers and amounted for the BG group $4.3 \%$ and for the HGH group $4.9 \%(p=0.598)$. Data is summarized in Table 2.

\section{Discussion}

Nearly 40 years after establishing the concept of ATLS, Mohammad et. al have shown in a review that ATLS significantly improves the knowledge of participants managing multiple trauma patients [2]. To evaluate the efficacy of standardized approach in different settings, in this study large collections of pelvic injury patients with different epidemiologic data were compared. On the one side the population of the BG group was generally older and there was almost the same amount of male and female patients. On the other side, the population of the HGH group mainly consisted of young males. In accordance to the different groups, 
the main trauma mechanism causing pelvic fracture differs matching the structure of the population; The patients from the HGH group mainly suffered from a road traffic related, whereas patients from the BG group mainly fall related (not regarding the height of the fall). The reason for this may be given by age-dependent living conditions. Young males are overrepresented in road crashes around the world [3-5]. As described in literature, mainly older people get actually injured following a fall from standing height $[6,7]$.

Despite the trauma mechanism an - although not significant - tendence to higher ISS values in the BG group could be found. Moreover, the average AIS pelvis was significantly higher in the BG group. Besides being hardly relevant from a clinical point of view, this difference is not solely explainable from the epidemiologic data. However, when regarding the classification of the injuries, patients in the BG group were more likely to suffer from a higherlevel injury (B-or C-type) what may explain the higher values in the AIS. Regarding the higher proportion of potentially osteopenic patients (elder females), the probability for a more severe rated pelvic injury can be expected to be elevated as well.

Regarding the primary outcome parameter, the incidence of complications during the inpatient stay, no statistically significant difference in the complication rate could be found. To the best of our knowledge, this is the first study to explicitly examine the frequency of typical complications of prolonged bedridding and ICU treatment.

The major part of these complications is made up by pulmonal affections. In the BG group there were significant more cases with the diagnosis of ARDS during posttraumatic ICU treatment. Furthermore, patients of the BG group have received significant more blood transfusions when they received any. A correlation in this observation might be, that allogenic blood transfusions can lead to lung injury [8,9]. Acute kidney failure was low in both groups and much lower that reported rates in literature that go up to $50 \%$ in critically injures trauma patients [10-13]. A reason for this discrepancy might be the inclusion of more stable non-ICU patients in this study and the early use of fluid resuscitation in trauma patients according to the ATLS protocol. None of the patient was in need of dialysis. Sepsis was as well observed in frequencies comparable to those in literature [14,15].

Regarding mortality the observed rates were comparable low with $4.3 \%$ and $4.9 \%$ respectively. Mortality rates of pelvic injuries vary in literature from comparable low rates up to around $60 \%$ mainly depending on accompanying injuries [16-20]. In polytraumatized patients, overall mortality rates are reported around 20\% in Germany [21]. There are several studies showing the effect of optimizing trauma care in reduced mortality rates [22-25]. Regarding the fact, that in this analysis, all patients were included, not only the ones that met polytrauma criteria, the observed mortality rates seem reasonable.

\section{Limitations}

One limitation is the retrospective design which might influence the generalizability of our results with some missing variables. Furthermore, preclinical data was not available from all patients, so there might be a bias due to limited data.

\section{Conclusions}

With the comparison of two Level-1 Trauma centers in very different countries with a very different population, it was possible to demonstrate that by implementation of priority-based ATLS, strategies in trauma management. Morbidity and mortality could be kept comparably low in patients with traumatic pelvic injury.

\section{Abbreviations}

$\begin{array}{ll}\text { ATLS } & \text { Advanced Trauma Life Support } \\ \text { ISS } & \text { Injury Severity Score } \\ \text { AIS } & \text { Abbreviated Injury Score } \\ \text { VAP } & \text { Ventilator associated Pneumonia } \\ \text { ARDS } & \text { Acute Respiratory Distress Syndrome }\end{array}$



ICU
Intensive Care Unit
CT
Computed Tomography

\section{Declarations}

Ethics approval and consent to participate: Ethics approval was given by local ethics committees in Germany and Qatar as stated above. As analysis was anonymized and retrospective, individual consent of the patients was not necessary.

Consent for publication: Not applicable

Availability of data and materials: The datasets used and/or analysed during the current study are available from the corresponding author on reasonable request

Competing interests: The authors declare that they have no competing interests

Funding: No funding was received for this study

Author's contributions: HK wrote the manuscript, JD and FW gathered the data of BG patients, did the analysis and created figures, AEM, HA, AA, SIG, EB and HAT gathered data of $\mathrm{HGH}$ patients, BMW, PAG and MM supervised the study and proof-read the manuscript. All authors read and approved the final manuscript.

Acknowledgements: none 


\section{References}

1. Gwinnutt CL, Driscoll PA. Advanced trauma life support. Eur J Anaesthesiol [Internet]. 1996 [cited 2019 Feb 7];13:95-101. Available from:

http://www.ncbi.nlm.nih.gov/pubmed/8829950

2. Mohammad A, Branicki F, Abu-Zidan FM. Educational and clinical impact of Advanced Trauma Life Support (ATLS) courses: a systematic review. World J Surg [Internet]. 2014 [cited 2019 Feb 7];38:322-9. Available from: http://link.springer.com/10.1007/s00268-0132294-0

3. Chen H, Chen Q, Chen L, Zhang G. Analysis of risk factors affecting driver injury and crash injury with drivers under the influence of alcohol (DUI) and non-DUI. Traffic Inj Prev [Internet]. 2016 [cited 2019 Feb 8];17:796-802. Available from:

http://www.ncbi.nlm.nih.gov/pubmed/27064506

4. Scott-Parker B, Oviedo-Trespalacios O. Young driver risky behaviour and predictors of crash risk in Australia, New Zealand and Colombia: Same but different? Accid Anal Prev [Internet]. 2017 [cited 2019 Feb 7];99:30-8. Available from:

https://linkinghub.elsevier.com/retrieve/pii/S0001457516303931

5. Abegaz T, Berhane Y, Worku A, Assrat A, Assefa A. Effects of excessive speeding and falling asleep while driving on crash injury severity in Ethiopia: A generalized ordered logit model analysis. Accid Anal Prev [Internet]. 2014 [cited 2019 Feb 8];71:15-21. Available from: http://www.ncbi.nlm.nih.gov/pubmed/24866353

6. Baidwan NK, Naranje SM. Epidemiology and recent trends of geriatric fractures presenting to the emergency department for United States population from year 2004-2014. Public Health [Internet]. 2017 [cited 2019 Feb 8];142:64-9. Available from:

http://www.ncbi.nlm.nih.gov/pubmed/28057200

7. Sherrington C, Fairhall N, Kirkham C, Clemson L, Howard K, Vogler C, et al. Exercise and fall prevention self-management to reduce mobility-related disability and falls after fall-related lower limb fracture in older people: protocol for the RESTORE (Recovery Exercises and STepping On afteR fracturE) randomised controlled trial. BMC Geriatr [Internet]. 2016 [cited 2019 Feb 8];16:34. Available from: http://www.ncbi.nlm.nih.gov/pubmed/26838998

8. Serpa Neto A, Juffermans NP, Hemmes SNT, Barbas CS V, Beiderlinden M, Biehl M, et al. Interaction between peri-operative blood transfusion, tidal volume, airway pressure and postoperative ARDS: an individual patient data meta-analysis. Ann Transl Med [Internet]. 2018 [cited 2019 Feb 7];6:23. Available from: http://atm.amegroups.com/article/view/18167/18474

9. Rezoagli E, Fumagalli R, Bellani G. Definition and epidemiology of acute respiratory distress syndrome. Ann Transl Med [Internet]. 2017 [cited 2019 Feb 7];5:282-282. Available from: http://www.ncbi.nlm.nih.gov/pubmed/28828357

10. Eriksson M, Brattström O, Mårtensson J, Larsson E, Oldner A. Acute kidney injury following severe trauma. J Trauma Acute Care Surg [Internet]. 2015 [cited 2019 Feb 8];79:407-12. Available from: http://www.ncbi.nlm.nih.gov/pubmed/26307873

11. Harrois A, Libert N, Duranteau J. Acute kidney injury in trauma patients. Curr Opin Crit Care [Internet]. 2017 [cited 2019 Feb 8];23:447-56. Available from: http://insights.ovid.com/crossref?an=00075198-201712000-00002

12. Gomes E, Antunes R, Dias C, Araújo R, Costa-Pereira A. Acute kidney injury in severe trauma assessed by RIFLE criteria: a common feature without implications on mortality? 
Scand J Trauma Resusc Emerg Med [Internet]. 2010 [cited 2019 Feb 8];18:1. Available from: http://sjtrem.biomedcentral.com/articles/10.1186/1757-7241-18-1

13. Harrois A, Libert N, Duranteau J. Acute kidney injury in trauma patients. Curr Opin Crit Care [Internet]. 2017 [cited 2019 Feb 8];23:447-56. Available from: http://www.ncbi.nlm.nih.gov/pubmed/29035925

14. Lalwani S, Rajkumari N, Mathur P, Sharma V, Trikha V. Sepsis in fatal pelvic trauma patients: report from a level-1 Indian Trauma Centre. Eur J Trauma Emerg Surg [Internet]. Springer Berlin Heidelberg; 2016 [cited 2019 Feb 8];42:43-5. Available from: http://link.springer.com/10.1007/s00068-015-0496-x

15. Lakomkin N, Sathiyakumar V, Wick B, Shen MS, Jahangir AA, Mir H, et al. Incidence and predictive risk factors of postoperative sepsis in orthopedic trauma patients. J Orthop Traumatol [Internet]. 2017 [cited 2019 Feb 8];18:151-8. Available from: http://www.ncbi.nlm.nih.gov/pubmed/27848054

16. Dente CJ, Feliciano D V, Rozycki GS, Wyrzykowski AD, Nicholas JM, Salomone JP, et al. The outcome of open pelvic fractures in the modern era. Am J Surg [Internet]. 2005 [cited 2019 Jan 17];190:830-5. Available from:

http://linkinghub.elsevier.com/retrieve/pii/S0002961005006793

17. Arvieux C, Thony F, Broux C, Ageron F-X, Rancurel E, Abba J, et al. Current management of severe pelvic and perineal trauma. J Visc Surg [Internet]. 2012 [cited 2019 Feb 8];149:e227-38. Available from: http://www.ncbi.nlm.nih.gov/pubmed/22818970

18. Rothenberger DA, Fischer RP, Strate RG, Velasco R, Perry JF. The mortality associated with pelvic fractures. Surgery [Internet]. 1978 [cited 2019 Feb 8];84:356-61. Available from: http://www.ncbi.nlm.nih.gov/pubmed/684627

19. Grotz MRW, Allami MK, Harwood P, Pape HC, Krettek C, Giannoudis PV. Open pelvic fractures: epidemiology, current concepts of management and outcome. Injury [Internet]. 2005 [cited 2019 Feb 8];36:1-13. Available from:

http://www.ncbi.nlm.nih.gov/pubmed/15589906

20. Hermans E, Edwards MJR, Goslings JC, Biert J. Open pelvic fracture: the killing fracture? J Orthop Surg Res [Internet]. 2018 [cited 2019 Feb 8];13:83. Available from: http://www.ncbi.nlm.nih.gov/pubmed/29653551

21. Ruchholtz S, Lefering R, Paffrath T, Oestern HJ, Neugebauer E, Nast-Kolb D, et al. Reduction in mortality of severely injured patients in Germany. Dtsch Arztebl Int [Internet]. Deutscher Arzte-Verlag GmbH; 2008 [cited 2019 Feb 8];105:225-31. Available from: http://www.ncbi.nlm.nih.gov/pubmed/19629200

22. Shafi S, Nathens AB, Elliott AC, Gentilello L. Effect of Trauma Systems on Motor Vehicle Occupant Mortality: A Comparison Between States With and Without a Formal System. J Trauma Inj Infect Crit Care [Internet]. 2006 [cited 2019 Feb 8];61:1374-9. Available from: http://www.ncbi.nlm.nih.gov/pubmed/17159679

23. Nathens AB, Jurkovich GJ, Cummings $P$, Rivara FP, Maier R V. The effect of organized systems of trauma care on motor vehicle crash mortality. JAMA [Internet]. 2000 [cited 2019 Feb 8];283:1990-4. Available from: http://www.ncbi.nlm.nih.gov/pubmed/10789667

24. MacKenzie EJ, Rivara FP, Jurkovich GJ, Nathens AB, Frey KP, Egleston BL, et al. A National Evaluation of the Effect of Trauma-Center Care on Mortality. N Engl J Med [Internet]. 2006 [cited 2019 Feb 8];354:366-78. Available from: http://www.ncbi.nlm.nih.gov/pubmed/16436768 
25. Radomski M, Zettervall S, Schroeder ME, Messing J, Dunne J, Sarani B. Critical Care for the Patient With Multiple Trauma. J Intensive Care Med [Internet]. 2016 [cited 2019 Feb 8];31:307-18. Available from: http://www.ncbi.nlm.nih.gov/pubmed/25673631 


\begin{tabular}{|c|c|c|c|}
\hline & & $\begin{array}{l}\text { BG group } \\
\text { amount (ratio) }\end{array}$ & $\begin{array}{l}\text { HGH group } \\
\text { amount (ratio) }\end{array}$ \\
\hline Age [years] & & 57.35 & 33.58 \\
\hline \multirow[t]{2}{*}{ Gender } & male & 357 (55,3\%) & 701 (88,6\%) \\
\hline & female & 288 (44,7\%) & $90(11,4 \%)$ \\
\hline \multirow[t]{2}{*}{ Nationality } & locals & $636(98,6 \%)$ & $119(15,0 \%)$ \\
\hline & foreigners & $9(1,4 \%)$ & $672(85,0 \%)$ \\
\hline \multirow[t]{4}{*}{ Trauma mechanism } & road traffic & $258(40,0 \%)$ & $471(59,5 \%)$ \\
\hline & fall & 355 (55\%) & 237 (30\%) \\
\hline & pedestriana & $28(4,3 \%)$ & $59(7,5 \%)$ \\
\hline & other & $4(0,6 \%)$ & $24(3 \%)$ \\
\hline \multirow{4}{*}{$\begin{array}{l}\text { AO classification pelvic } \\
\text { fracture }\end{array}$} & no fracture & $95(14,7 \%)$ & $148(19,7 \%)$ \\
\hline & $61 \mathrm{~A}$ & $238(36,9 \%)$ & $423(56,3 \%)$ \\
\hline & $61 \mathrm{~B}$ & $238(36,9 \%)$ & $108(14,4 \%)$ \\
\hline & $61 \mathrm{C}$ & 74 (11,5\%) & $73(9,7 \%)$ \\
\hline \multirow[t]{2}{*}{ Pelvic operation } & Yes (OM) & $226(35,0 \%)$ & $361(45,6 \%)$ \\
\hline & No (NOM) & $419(65,0 \%)$ & $430(54,4 \%)$ \\
\hline
\end{tabular}

Table 1: Descriptive analysis 


\begin{tabular}{llll}
\hline & $\begin{array}{l}\text { BG group } \\
\text { amount (ratio) }\end{array}$ & $\begin{array}{l}\text { HGH group } \\
\text { amount (ratio) }\end{array}$ & p-value \\
\hline VAP & $31(4,8 \%)$ & $56(7,1 \%)$ & 0,072 \\
Acute kidney failure & $22(3,4 \%)$ & $20(2,5 \%)$ & 0,324 \\
ARDS & $36(5,6 \%)$ & $14(1,8 \%)$ & 0,000 \\
Sepsis & $20(3,1 \%)$ & $26(3,3 \%)$ & 0,842 \\
\hline
\end{tabular}

Table 2: Complication rates 


\section{Figure legends}

Figure 1: Distribution of initial AIS as on presentation in trauma room

Figure 2: Distribution of initial ISS as on presentation in trauma room 
Figures

\section{Image not available with this version}

Figure 1

Distribution of initial AIS as on presentation in trauma room

\section{Image not available with this version}

Figure 2

Distribution of initial ISS as on presentation in trauma room 